\title{
Unsere Kinder, unsere Zukunft
}

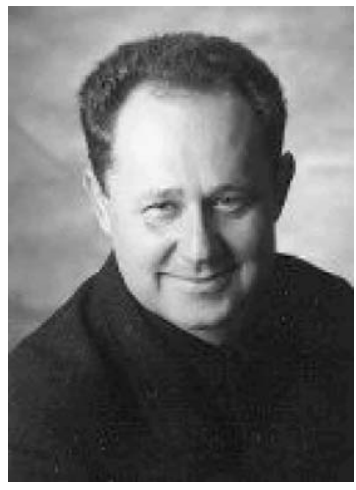

Prof. Dr. Manfred Wildner
Bibliografie

DOI http://dx.doi.org/ 10.1055/s-0031-1286255

Gesundheitswesen 2011;

73: 467-468

(c) Georg Thieme Verlag KC

Stuttgart · New York

ISSN 0941-3790

Korrespondenzadresse

Prof. Dr. M. Wildner

Bayerisches Landesamt

für Gesundheit und

Lebensmittelsicherheit

Veterinärstraße 2

85762 Oberschleißheim

Manfred.Wildner@|gl.bayern.de
Das Wissen über Nikomachos, den Sohn des Aristoteles, ist begrenzt. Ob Aristoteles tatsächlich seinem Sohn eine bedeutende Schrift - die „Nikomachische Ethik“ - gewidmet hat, ist umstritten [1]. Die Vorstellung, dass er dies tat, um so Nikomachos den Weg zu einem gelungenen Leben zu weisen, würde auf ein liebevolles, um gegenseitige Verantwortung wissendes Verhältnis zwischen den Generationen hinweisen. Solches Handeln ist auch in der Moderne zu finden, wie die kleine „Ethik für die Erwachsenen von morgen" Fernando Savaters oder Jackson Browns „Life's little instruction book“ zeigen [2,3]. Dass „Zank und Streit eben daher [kommen], dass entweder Gleiche nicht Gleiches oder nicht Gleiche Gleiches bekommen und genießen“, ist eine der wesentlichen Einsichten der Nikomachischen Ethik [4]. Aristoteles unterscheidet dabei den Ansatz der Ergebnisgerechtigkeit, nämlich dass alle Mitglieder einer Gesellschaft an einem Gut grundsätzlich in gleichem Maße teilhaben sollten, soweit nicht eigenes Verschulden vorliegt, von der Regelgerechtigkeit. Letztere bezeichnet die faire Anwendung von vereinbarten Regeln und das bedeutet eben, dass Gleiche gleich und Ungleiche ungleich behandelt werden sollten. Mit etwas Augenzwinkern: Ob kindlicher Streit zwischen Nikomachos und seiner älteren Schwester Pythias der Ausgangspunkt für Aristoteles' tiefgreifende Reflexionen war?

Ein Thema für das Gesundheitswesen? Gewiss: Fragen der Verteilungsgerechtigkeit dürften vor dem Hintergrund von Rationierungs- und Priorisierungsdiskussionen zunehmend an Relevanz gewinnen. Dies betrifft das Ergebnis der Verteilung ebenso wie die prozeduralen Regeln. Was im Gesundheitswesen verteilt wird? Eine ganze Menge: Zugang zu medizinischen Leistungen und Rangplätze auf Transplantationslisten, Intensivbetten und Operationssäle, Wartezeiten und angerechnete Beschäftigungsverhältnisse, Lohnprozentprämien und Zuzahlungen, Kassenarztsitze und Punktwerte, Regelleistungsvolumina u.v.a.m. In monetären Einheiten hunderte von Milliarden Euro, in letzter Konsequenz Gesundheitschancen, Lebensqualität und Lebenserwartung. Haben wir nicht genug für alle? Dass die Raffgier Einzelner wohlgeordnete Sozialsysteme schädigen und zerstören kann, ist eine bekannte Tatsache - in der Ökonomie z.B. als „AllmendenProblem“ oder „moral Hazard“ bekannt. „Die Welt hat genug für jedermanns Bedürfnisse, aber nicht für jedermanns Gier“, lautet ein Mahatma Gandhi zugeschriebenes Zitat, welches einen Teil der beobachteten Verknappung erklären dürfte.
Wo diese, auch Sozialversicherungssysteme zerstörende Gier, anzutreffen ist? Auf Seiten der Versicherten bei Sozialbetrug und Leistungserschleichung bzw. grundloser Inanspruchname „kostenfreier“ Leistungen, bei Schwarzarbeit und Bestechung. Bei den Leistungserbringern bei Abrechnungsbetrug und DRG-Upcoding, bei unangemessener angebotsinduzierter Nachfragesteigerung, bei Leistungsverschiebungen und -verweigerungen, bei Über-, Unter- und Fehlversorgung und Fahrlässigkeit. Zudem wird wohl auch „am großen Rad“ gedreht: von Investmentgesellschaften nicht nur mit Heuschrecken-Taktiken, sondern auch mit unbalancierter „shareholder value“-Doktrin zu Lasten von Patienten und Mitarbeitern, durch Gewinnmaximierung und -abschöpfung ohne nachhaltige Reinvestitionen. Dass zu viel Markt nicht gesund macht, ist bereits kommentiert worden, ebenso, dass sich das Armutsproblem zunehmend auch als Reichtumsproblem erweist [5-7]. Ist Vermögen im Vergleich zum Arbeitslohneinkommen hinreichend besteuert oder bezahlt die Sekretärin für den Millionär, der Lackierer für den Chefarzt? Es wird inzwischen schon von einer massiven „Unwucht zwischen Realwirtschaft und Finanzwirtschaft" gesprochen - als Schicksals- und Friedensfrage. Das Verhältnis von materiellem Vermögen und dem Vermögen des Geistes und der Arbeitskraft sei neu zu bestimmen: nicht Kapital arbeitet, sondern der Mensch [6].

Unsere Sozialversicherungssysteme und das Gesundheitswesen mit ihnen haben eine nur noch schwer zu durchschauende und zu steuernde Komplexität erreicht. Ärztliche Ethik allein - so wichtig und unverzichtbar diese auch ist - genügt nicht mehr als Handlungsleitfaden für das System. Traditionelle ärztliche Ethik ist individualmedizinisch ausgerichtet. Sie will als Berufsethik vor allem die Arzt-Patientenbeziehung und die Beziehungen innerhalb der ärztlichen Profession ordnen. Damit ist sie zeitlichem und kulturellem Wandel ausgesetzt: während der Hippokratische Eid noch das professionelle innerärztliche Verhältnis an den Anfang setzt, setzt das Genfer Ärztegelöbnis den Dienst an der Menschlichkeit an die erste Stelle - ein durchaus bemerkenswerter Schritt [8]. Dieses Gelöbnis gilt in Deutschland in leicht modifizierter Form für alle Ärzte bei Aufnahme in den Berufsstand. Sie ist Teil der (Muster-)Berufsordnung mit einer Vielzahl an einzelnen Artikeln, welche der gesellschaftlichen Entwicklung Rechnung tragen wollen. Über die ärztlichen Pflichten gegenüber den Patientinnen und Patienten hinaus, wie dem 
Schutz anvertrauter Privatgeheimnisse, sind auch neue (System-)Themen wie die Qualitätssicherung, das Verhältnis zur Industrie oder die Berufsausübung im Rahmen der EU angesprochen [9]. Dass alle Ärzte und Ärztinnen bei der Ausübung ihrer ärztlichen Pflichten „keinen Unterschied machen weder nach Religion, Nationalität, Rasse noch nach Parteizugehörigkeit oder sozialer Stellung“, wie im Genfer Ärztegelöbnis versprochen, bleibt zu hoffen. Dass die Thematik sexuellen Missbrauchs unter Ausnutzung eines Beratungs-, Behandlungs- oder Betreuungsverhältnisses anders als im ursprünglichen hippokratischen Eid nicht mehr explizit erwähnt ist, ist vielleicht dadurch zu erklären, dass der Gesetzgeber einen entsprechenden Paragrafen in das Strafgesetzbuch eingeführt hat (§174c).

Die genannten, auf das ärztliche Handeln fokussierten, Grundsätze sind dabei nicht ausreichend, um (Verteilungs-)Gerechtigkeit auf gesellschaftlicher Ebene zu sichern: Institutionen- und ordnungsethische Beiträge sind notwendig [10]. Der dritte Armuts- und Reichtumsbericht hält fest: Kern sozial gerechter Politik ist es, ökonomische und soziale Teilhabe- und Verwirklichungschancen für alle Mitglieder in der Gesellschaft zu ermöglichen [7, S. 12]. Ein wichtiger Eckpunkt dieser Debatte ist der Gleichheitssatz des Verfassungsrechts: jus respicit aequitatem das Recht achtet auf Gleichheit. Aus dem Naturrecht kommend, ist dieser Grundsatz sowohl im Europäischen Recht (Artikel 12 und 141 des EG-Vertrages sowie die EU-Grundrechtecharta) als auch im deutschen Grundgesetz verankert (Artikel 3). In der gesundheitswissenschaftlichen Diskussion wird dabei die Gleichheit im gesundheitlichen Zustand (equality), welche für Bevölkerungen irreal ist, von der gesundheitlichen Chancengleichheit (equity) unterschieden. Letztere ist ein wichtiges gesundheitspolitisches Ziel. Es sollte nicht nur einer unsichtbaren Hand überlassen werden, sondern ist zumindest in der europäischen (Werte-)Union auch ein wichtiges Ziel der ordnenden Funktionen der öffentlichen Hand [11]. Hinzu kommen Fragen der intergenerationalen Gerechtigkeit, also der Verantwortlichkeiten zwischen der Eltern- und Kindergeneration. Betrifft der „Generationenvertrag“ wirklich nur mehr Eltern und Kinder, oder sind hier Dritte und Vierte dazugekommen, während Zweite fehlen? Gibt es nicht auch noch die langlebigen Großeltern, ein „Drittes“ und „Viertes“ Alter, also mehr als eine Generation in der Phase des Posterwerbslebens? Und zu wenige Mitglieder auf der Vertragsseite der Kinder, welche die Erfüllung der Erwartungen übernehmen soll? Es scheint, dass der Generationenvertrag hier der Nachbesserung bedarf, um eine sich entwickelnde Schieflage im berechtigten Interesse der Kindergeneration zu korrigieren.

Wie dieses Ziel nachhaltig erreicht werden kann, unter Berücksichtigung der Gesundheit unserer Kinder, ist direkt oder indirekt Gegenstand der verschiedenen Beiträge in diesem Heft: es geht um perinatale Mortalität, um ein universelles Neugeborenen-Hörscreening, um Zahngesundheit bei Erstklässlern, um Helmtragequoten bei Jugendlichen und Strategien zur Verbesserung von Impfraten, um die Zulassung und Erstattung sog. „Orphan Drugs“, um Gesundheitsmanagement in kleineren und mittleren Unternehmen und um Qualitätsfragen der Begutachtung in der gesetzlichen Sozialversicherung - ein wichtiges Instrument (verteilungs-)gerechter Leistungsgewährung.

Wie Nikomachos auf das „theoretische“ Geschenk seines Vaters wohl reagiert hat? Vielleicht wäre ihm ein gesunder Kapitalstock (nicht nur) für seine Altersversorgung lieber gewesen. Ego, hic, nunc - Kinder leben zunächst vor allem bezogen auf sich selbst, den eigenen Standpunkt, das Jetzt. Vielleicht hat Aristoteles dies bei seinem Geschenk schon bedacht und auch, dass die zukünftige Gegenwart der Kinder ganz anders aussehen könnte als die gegenwärtige Zukunft, die sich die Eltern vorstellen. Auf lange Sicht und bezogen auf gesellschaftliche Fragen war die vertiefte Diskussion um (Verteilungs-)Gerechtigkeit, die sein Vater begonnen hat, sicherlich nachhaltiger als die augenblickliche Anhäufung von Ressourcen. Gleiches mag auch für uns gelten.

\section{Literatur}

1 Aristoteles' Nikomachische Ethik (Übers. Eugen Rolfes). Verlag Felix Meiner, Leipzig, 1911. URL: http://gutenberg.spiegel.de/buch/2361/1 Zugriff am 07.08.2011

2 Savater F. Tu was du willst. Ethik für die Erwachsenen von morgen. Campus Verlag, Frankfurt 2007

3 Brown HJ. Life's little instruction book. Rutledge Hill Pr Nashville, Tennessee, 1994

4 Aristoteles Nikomachische Ethik: Fünftes Buch, sechstes Kapitel (Übers. Eugen Rolfes). Verlag Felix Meiner, Leipzig 1911. URL: http:// gutenberg.spiegel.de/buch/2361/1 Zugriff am 07.08.2011

5 Hengsbach F. „Mehr Markt“ macht nicht gesund - Gesellschaftliche Risiken und solidarische Sicherung entsprechen einander. Gesundheitswesen 2008; 70: 339-349

6 Kommentar: Jung und reich. Christ in der Gegenwart 2011; 30: 329

7 Deutscher Bundestag, Lebenslagen in Deutschland - Dritter Armutsund Reichtumsbericht. DS 16/9915 vom 30.06.2008. URL: http:// dip21.bundestag.de/dip21/btd/16/099/1609915.pdf Zugriff am 08.08.2011

8 Weltärztebund. Deklaration von Genf 1948/1968/1983/1994. URL: http://www.bundesaerztekammer.de/downloads/Genf.pdf Zugriff am 08.08.2011

9 Bundesärztekammer. (Muster-) Berufsordnung für die deutschen Ärztinnen und Ärzte. 2006. URL: http://www.bundesaerztekammer.de/ downloads/MBOStand20061124.pdf Zugriff am 08.08.2011

10 Kuhn J, Wildner M. Ethik in der Gesundheitsförderung und Prävention. In: Bundeszentrale für gesundheitliche Aufklärung, Hrsg. Leitbegriffe der Gesundheitsförderung und Prävention. Verlag für Gesundheitsförderung, Gamburg, 2011; S. 65-S. 68

11 Europäische Gesundheitsministerkonferenz der Weltgesundheitsorganisation Europa. Die Charta von Tallinn: Gesundheitssysteme für Gesundheit und Wohlstand. Tallinn, Estonia, 27. Juni 2008 http:// www.euro.who.int/document/e91438g.pdf Zugriff am 02.01.2009 